\title{
IDEAH
}

\section{Rolling the Dice on Project Management}

\section{Quinn Dombrowski ${ }^{1}$}

\section{${ }^{1}$ Stanford U}

Published on: Nov 25, 2021

License: Creative Commons Attribution 4.0 International License (CC-BY 4.0). 
Over the last decade, there has been a growing recognition of the importance of good project management practices in the context of digital humanities projects. While project management tasks have often fallen to graduate student assistants who are put in a position of figuring out project management as they go, formal training in project management is now commonly offered at workshops and summer institutes. $\underline{1}$ These short-term learning opportunities serve as a valuable crash course in some core concepts, terminology, and procedures of project management, but as a consequence of their limited duration, there is little space for students to grapple with the realworld complexity of what it would look like to apply these procedures in the context of a real project. This paper advocates for an expansion of the typical project management curriculum-even at the expense of other material-to include simulations, or similar activities that require students to grapple with the complexity of project management in the messy context of people's real lives. It takes as a case study DLCL 205, "Project Management and Ethical Collaboration for Humanists", a onequarter course offered at Stanford University in winter 2020. As one of the digital humanities courses in the Division of Literatures, Cultures, and Languages (DLCL), DLCL 205 was conceptually paired with a hands-on, technically-oriented course that taught practical skills for doing text-based digital humanities $(\mathrm{DH})$ work in languages other than English. However, in many projects technology itself is not the decisive factor in project success or failure: rather, project management and relationship management are more likely to determine the project's outcome. While these issues were a cornerstone of class discussion, the course also provided students with an environment for experimenting with different choices and experiencing the consequences of those choices without negative real-world effects. This environment was the Digital Humanities Roleplaying Game (DH RPG), a tabletop role-playing game in the style of Dungeons and Dragons(D\&D), where each student played a role (undergraduate, graduate, postdoc, staff, librarian, or untenured principal investigators (PIs)) who would play some role in a year-long DH project.

The course operated from the premise that focusing on documents (e.g., project charters, budgets, scopes, and work plans) and processes (e.g., team-building, communication methods, and holding effective meetings) provides students with a useful toolkit. However, such project management curricula implicitly oversell the toolkit's impact by not discussing the systemic inequalities in the contexts where the toolkit will be deployed. This is a large and complex issue, and not one amenable to a brief discussion in a half-day or even week-long workshop. While recent scholarship on 
the systems of higher education have begun to broach these issues (consider Fitzpatrick 2019, Thinking Generously), it is still rare to acknowledge them publicly, let alone grapple with their impact on one's scholarship, mentoring, teaching, and collaboration in academic contexts. A quarter-long course is a more reasonable period of time to undertake serious consideration of roles, power, and incentives in the academy, particularly when these issues are raised in a practical, applied context, rather than with the psychological distance afforded by a theory-oriented approach. While time constraints are a significant impediment to directly adopting the framework used by this class, this paper may nonetheless shed some light on a broader context that DH project managers will inevitably encounter when they attempt to apply what they have learned and may also provide some inspiration for reimagining project management curricula.

\section{Games and gaming in DH pedagogy}

There is a long and rich history of incorporating games into the DH curriculum. Early examples include the Ivanhoe game of literary criticism (Drucker and Rockwell 2003) in the early 2000's, reimagined a decade later by two Scholars Lab Praxis Fellows cohorts into a WordPress theme (ivanhoe.scholarslab.org) that was adopted in contexts such as Genelle Gertz’s course on Milton (Gertz 2015). In “A Long-Belated Welcome: Accepting Digital Humanities Methods into Non-DH Classrooms", Kara Kennedy gestures towards gaming (including activities such as modeling historical spaces in Second Life) as a DH method that could be fruitfully extended to non-DH classrooms. For the Digital Humanities Summer Institute (DHSI) 2015 workshop "Models for DH at Liberal Arts Colleges", Gregory Lord, Angel David Nieves, and Janet Simons developed dhQuest, a game based on the tabletop role-playing game Dungeons \& Dragons, but "meant to model the process of beginning a Digital Humanities center at an institution, and the many (many) decisions that are involved along the way" (Lord et al 2016). The game was also available in a Unity-based online version, and source code is available on GitHub. $\underline{2}, \underline{3}$ In 2019, students in Shawn Graham's DIGH5000 graduate course at Carleton University created DH Unplugged, an analog card game where players are part of a think tank "engag[ed] in creative problem-solving for local and global problems", drawing on resources (represented by cards) such as digital technologies, raw materials, and art. $\underline{4}$

dhQuest and DH Unplugged both model DH as the accumulation of the right mix of resources (be they technical / material cards in DH Unplugged, or spending things like time and funding to accrue credibility and networks in dhQuest). $\underline{5}$ The DH RPG used in 
DLCL 205 lacked a gameplay element around resources, instead structuring the game around time and labor. Credibility or networks aren't discovered in treasure chests: they're the product of time spent on activities with social payoff. dhQuest models this through individual "quests" that can accrue credibility or network points in exchange for resources like time, staff, and institutional support. Not all roles have the same number of each resource type for each turn, and dice rolls during the character creation process create space for additional, realistic variation between characters: not everyone is in a position to spend time on activities that build network or credibility, given other constraints and obligations in their life. dhQuest creates a straightforward, transactional system for quest completion: having the right number of resources means the quest will be completed successfully. If a player chooses to undertake quest \#13: "attend a conference related to your field", the only possible outcome is:

You decide to invest a bit of time and money toward your own development, in the hopes that rubbing elbows with your colleagues might lead to new opportunities and a bit of useful inspiration in your work. The effort pays off nicely, as you now have a new list of interesting and valuable names from your field--and hopefully a few of them now have yours as well.

There is no possibility of accidentally insulting a senior scholar, spilling wine on oneself, or missing most of the conference due to gastrointestinal distress. In the real world, dedicating resources to activities meant to build network or credibility does not mean that it will be a success. dhQuest incorporates surprise and randomness into each turn with a "But suddenly..." message at the end of the quest outcome description; this is a randomly-chosen event with some resource bonus (e.g., IT upgrades your computer) or penalty (one of your DH allies leaves for graduate school). The predictable occurrence of a surprise, plus the fact that it is not linked to the content of the quest that it follows, lessens its impact. By replacing abstracted "resources" with simply time and labor, determining power and privilege through players' character sheets, and making luck and dice rolls an integral part of taking any action, the DH RPG surfaces institutional and interpersonal factors at play - often silently-in DH projects, to an extent uncommon in DH pedagogical games.

\section{Challenges for ethical collaboration}

It is well known that undergraduates have a very poor understanding of university titles, roles, and their hierarchies, leading to surprise and dismay when they discover their favourite "professor" is an adjunct whose contract won't be renewed, or a grad 
student whose labor conditions have led to them going on strike. While these kinds of basic role distinctions are usually not lost on $\mathrm{PhD}$ students, the clarity of their understanding of how the university works drops off quickly as soon as the roles branch out (e.g., to library and staff positions) or up (e.g., responsibilities of department chair vs. dean vs. provost). There is little incentive to provide graduate students with a comprehensive overview of the system as part of basic professionalization classes or workshops. Giving students a view into where different levers of power are located in the university can be seen as inviting trouble. Without an understanding of how the systems of the university operate, students' ability to do anything from accomplishing administrative tasks to enacting large-scale change depends on who they know, and the goodwill of faculty, librarians, and staff who they encounter. While a tenure-track job is presented as the highest reward for "successfully" completing graduate school, graduate students may have a dim understanding of the actual living and working conditions that come with such a position, or the sacrifices that junior faculty make with regard to various other priorities in their lives (e.g., choice of where to live, whether or when to have children, etc.). Depending on their advisor's position, they may get a glimpse of this reality in unguarded moments - but here, too, junior faculty may see it as not in their interest to complain or take any action that might dissuade graduate students from the proscribed path. For faculty who do take proactive steps to encourage graduate students to pursue other paths, graduate students may question their motives: whether it comes from a place of sincere concern and personal unhappiness, or if dissuading future competition (for jobs or $\mathrm{DH}$ funding) may be a factor.

Such a context is ripe for collaborations that verge on exploitation, or that may unambiguously cross that line - even without that intention. Ethical collaboration depends on an understanding of what's motivating the other participants. This may be straightforward and easily discernible (e.g., a student needs to make money and would rather proofread Optical Character Recognition (OCR) from home than shelve books at the library with a less-flexible schedule), or there may be reasons for project participants to conceal aspects of their motivation that they feel might jeopardize their relationships or standing within the group (e.g., a graduate student wanting to take the lead on developing a project with a guaranteed launch date and stable digital presence, in order to use it as part of a portfolio for pursuing non-academic employment over their advisor's objections).

Ideally, the ethical responsibilities of a project team extend beyond their responsibilities towards one another, and include considerations related to the 
materials they are working with, as well as any "invisible collaborators" whose labor is called upon through outsourcing components of the project work. These aspects of DH work are often overlooked by project PIs until someone points them out and are particularly invisible to students who are used to being the beneficiaries of processes. One such example is the library's Interlibrary Loan article request system, which appears to work as if by magic or automation, with no acknowledgement of the humans upon whom the whole system depends. To surface these issues, one class session brought in a guest speaker, Dinah Handel, the digitization services manager for Stanford Libraries. Readings including Robertson 2016a, Robertson 2016b, and Cowan and Rault 2018 complemented her presentation by raising issues that merit consideration and care with certain kinds of materials, including the materials at the center of the imaginary DH project that formed the starting scenario for the DH RPG as played in DLCL 205 in winter 2020. In this scenario, an assistant professor character (played by one of the students) had received a grant to digitize, publish online, and "do text analysis" (framed deliberately vaguely) on the full run of a collection of the Russian radical feminist lesbian zine Остров (Ostrov, "Island") from the 2000's and 2010's. $\underline{6}$ While these materials are generally less explicit and less personally-identifiable than those referenced by Robertson or Cowan and Rault, issues of privacy, consent for broad web-based distribution, and the risk of jeopardizing the safety of the contributors (particularly in a country with a recent history ranging from imprisonment to murder of sexual minorities) remain relevant.

Reaching a conclusion that it is ethical to digitize a particular set of materials is not the end of the ethical considerations with regard to digitization. As Handel noted, the question of how digitization will occur can also be fraught with ethical issues. To dispel students' assumptions that digitization is "easy work", Handel described some of the physical risks involved in doing digitization, particularly as a full-time job. Repeated stress injuries are not uncommon, and the work can involve spending long stretches of time in a darkened room near high-intensity light sources. Handel described a situation where she had to decide not to purchase a particular light whose technical specifications met the needs of the digitization's lab, but which staff found too painful to use. Ethical considerations in how to digitize materials can impact project timelines; when having sensitive materials (e.g., depictions of violence) digitized, it is more ethical to request a timeline that allows for staff to be rotated off that project, rather than being exposed to that material for days or weeks on end. To reinforce Handel's message about the physical toll of digitization, the course included a lab component where students took turns scanning pages of an issue of Остров on a flatbed scanner 
typical of those used by $\mathrm{DH}$ projects that do their own digitization. The students noted the physical discomfort of the chair and awkward arrangement of the computer and scanner, as well as the tedium of a task that nonetheless requires a moderate amount of focus to ensure quality results. This hands-on experience gave students pause later in the quarter when deciding how much time they wanted their DH RPG character to spend scanning materials.

\section{Empathy and imagination}

The makeup of the class provided numerous opportunities for students to serve as resources and reality checks for one another both during the class discussion of the readings and, in particular, during the DH RPG sessions. Officially, there were three students enrolled: an undergraduate, a master's student, and an early-stage $\mathrm{PhD}$ student. In practice, there were numerous auditors who attended with varying degrees of frequency, including multiple late-stage $\mathrm{PhD}$ students, a postdoc, and a librarian who had previously worked as tenure-track faculty. While every student played a different role than in real-life, having all roles represented through students' current or past experiences was invaluable. As the game moved through the year (typically a little under a month of "game time" for every class session), students' input informed the constraints that their peers' characters faced. The real-life postdoc was able to speak to the resources that were or were not in place for temporary members of a university community. The real-life librarian set benchmarks for how much time the ingame librarian would need to spend on her core job responsibilities-rather than fun add-ons to her workload like the DH project at hand-to keep faculty, students, and library administration satisfied at different points in the year (e.g., managing the influx of book reserve requests before the start of the quarter).

This arrangement imbued the experience with authenticity: rather than hearing from the instructor about the typical expectations from a subject-area librarian, the students heard it from someone currently holding that role and doing that work. It was, nonetheless, a risky pedagogical maneuver, where a positive outcome is dependent on a sense of camaraderie and support among the students. Some of this was simply a matter of good fortune in the particular mix of personalities in the class, but the framing of the DH RPG itself also played a role. While it quickly became evident to the students that in this game the value truly was in the journey rather than the destination, the initial presentation of the DH RPG as a "game" unavoidably leads to questions about "winning." In future iterations of the course, it may be better to reframe the task simply as a simulation, or something more broadly such as a 
"meaningful play activity." In an attempt to mitigate concerns about victory upfront, the one-page "getting started" handout put it this way:

In principle, everyone can "win" the DH RPG! Each character has 1-2 individual goals that they need to complete in addition to the project goals. Because the project goals have to be met for anyone to win the game, working together is essential.

The "getting started" materials for the DH RPG materials were also very direct about the linked goals of cultivating imagination and empathy, and how students should approach the game:

Imagination and empathy are at the heart of the DH RPG. For the RPG to be a meaningful simulation of a real DH project, you should use your imagination to try to embody the worldview of your character and make decisions that they would make within that worldview... At the same time, resist the temptation to descend into an exaggerated parody of your character type: you're playing a person, not a stereotype.

For purposes of this class, all players must play in good faith: you are actually trying to accomplish your character goals and the project goals, and shouldn't actively take malicious steps to sabotage the project or others' goals. At the same time, it may be realistic for your character to struggle with their goals and/or the project at some points in the game. Your character may have to deal with a family crisis or stop working on the project for a month to manage the impact of budget cuts on their primary job. In the real world, team members have all kinds of reactions to these sorts of situations, shaped by their own background, experience, and disposition. In the DH RPG as played in this class, empathy will be our lodestar. Players should modulate their characters' reactions with empathy to the extent possible. In this way, we play the world of DH not as it is, but as we'd like to see it.

\section{Time management for the DH RPG}

During the DH RPG segments of the course, each student has to play their character's turn in the world of the DH RPG. A turn consists of a player deciding what their character will do during that month by allocating their 20 activity points to different activities. 7 All characters have the same number of activity points, reflecting the twenty-four hours every person has in the day. Many activities-including those essential for completing project and character goals, like writing, teaching, and 
various technical tasks-require players to roll the dice to determine how successful their character was at doing the activity. Even if a player decides to spend 10 activity points - half of their allotment for the whole month - on writing, a bad dice roll may mean that very little actually got written (e.g., due to distraction or the cumulative effect of small acts of procrastination). As an activity, writing is particularly complex, involving two dice rolls: one to see how many words got written (using the Management skill group), and one to determine writing quality (using the Disciplinary skill group). Getting a high writing roll with a low quality roll accumulates a large number of "editing points", which must be removed (by trading one activity point to eliminate one editing point) before the written work is "complete." The number of dice that a player rolls to accomplish any particular activity varies based on the character's role, how the player has customized their character, as well as leveling up through experience gained in-game. These dice represent skill level differentials that can (but do not always) impact how well characters perform an activity; any player can have a bad dice roll regardless of role..

The game is directed by the DM (DH Master), a game master or referee who knows the game rules and narrates the events and surprises of each turn. 8 In DLCL 205, the DM was the instructor, but in other contexts, it may make more sense to team-teach a course or workshop, delegating the DM role to a librarian or staff member with extensive "in the trenches" experience with DH. Firsthand experience with the upsand-downs of DH projects-ideally, many kinds of DH projects, with many different types of collaborators - is important for a DM, and perhaps less so for the course instructor. The DM must be able to, at a moment's notice, come up with realistic surprises - good, bad, and ambiguous. At times, they may play auxiliary characters, like an unscrupulous contractor or a cantankerous sysadmin. 9 In DLCL 205, the DM also served as note-taker, keeping track of what happened in each turn, how much work was left to be done on various project tasks, and so on. This was challenging to maintain along with game management, and in future iterations, the role of "scribe" will rotate among players.

To illustrate how gameplay works in practice, imagine a player spends 5 activity points so that the graduate student they are playing can work on writing her dissertation. This chapter of the dissertation is going to take 20 writing points to complete. By default, a graduate student character has one die in Management (to determine their character's success at getting words written), and three dice in Disciplinary (to determine writing quality). This player has created a character who is extremely selfdisciplined, assigning some of the character customization points to Management, 
which allows them to roll three dice (instead of one) when rolling for writing. If the player's Management roll is above a certain threshold (let's say 12), they can apply all the activity points they dedicate to writing as writing points-meaning that 15 writing points would be left on the dissertation chapter. If the player hadn't customized their character by increasing their Management skill, it would be impossible for the player to get a 12 while rolling the default one die in Management. Even rolling three dice, it is possible to roll less than a 12. When a Management roll doesn't meet the defined threshold, the player gets fewer writing points than the number of activity points invested in writing-to factor in time lost to social media distractions, snack breaks, bored web surfing, and the like. There are different cut-offs for the character to get different amounts of writing points, based on the amount of time invested. Even if a player puts 10 or 15 activity points towards writing, shirking other responsibilities in the process, rolling a 2 for writing means that none of the activity points translate to writing points- which is much more likely for characters with one die in Management. Let's assume that the player rolls a 9 on writing, meaning that of the 5 activity points invested in writing, 4 of them translate into writing points, leaving 16 writing points remaining on the dissertation chapter. The player then rolls for quality. Let's say the player did not customize the character's Disciplinary skill, so they will roll the default three dice for graduate students. Writing is a complex task in real life; it is possible to successfully focus on writing, produce a lot of words, and realize that what you've written goes down a rabbit hole and is not actually useful for furthering your argument. The number of writing points the player has gained represents the maximum number of editing points they could potentially accumulate. Fortunately, it is mathematically impossible for the graduate student (with a Disciplinary roll of three dice) to roll a 2 , and acquire the 4 editing points that would correspond with their 4 writing points - this is possible for undergraduate characters with single die Disciplinary roll, whose character might, for instance, write a paper only to discover their major sources were actually linked to a conspiracy theory. Similar cutoffs apply to editing points as writing points. The player rolls a 10 this time and accrues one editing point on the dissertation chapter. They will need to exchange one activity point in the future to remove that editing point before they can submit the chapter. As an alternative, they could convince another character to edit it for them, and that player would have to spend the activity point.

Another key mechanism of the DH RPG is the "randomness die." Every time the players roll, one die is a different colour; If the player rolls only one die, they use the non-standard coloured die. If the differently-coloured die gets any number larger than 
1 , it counts on face value. If the player rolls a 1 on that die, the entire dice roll for that activity doesn't count, and the player must instead roll the 20-sided "randomness die." What happens next is left to the DM's discretion and imagination: a low roll on the D20 leads to something catastrophic, a high roll leads to something surprising but excellent, and a middle roll has no major impact but may derail the character's plans. In this way, a low score on the differently-coloured die does not necessarily spell doom, merely a loss of personal control, reflecting the fact that oftentimes things "just happen." All students in the class experienced the impact of the randomness die at various points throughout the quarter.

While the combined effect of these gameplay elements resulted in a set of character experiences that meaningfully resonated with the students who had lived through similar situations, the game moved slowly. The time required to play the game scales linearly with the number of players, and the timeline for the DH RPG as written in the syllabus assumed there would be no more than five students. Calculating the time required based on those assumptions, the syllabus claimed that the class would be able to progress through two months of game time in each class session, in order to traverse the entire year by halfway through the semester, debrief, and then play again.

The possibility for this "Groundhog Day" repetition of the whole RPG was inspired by Andrew P. Quick and Austin Zumbro's musical adaptation of Hervé Tullet's book Press Here - in which two unsuspecting "audience members" find themselves in a perplexing situation with a series of requests that they bungle, before getting the chance to repeatedly "do it all over again." The ability to re-encounter the same scenario with better preparation and wiser choices was fundamental to how the DH RPG was imagined, and how it was scheduled in the syllabus. The expectation was that the first time around, students would not give sufficient attention to the project management aspects of the project. As in real life, scholars often dismiss the value of project management the first time they do a project, and only adopt practices that draw upon project management tools and frameworks in response to challenges that arose as a result of ad-hoc management. Doing the DH RPG twice was necessary logistically because some important project management decisions (including naming a project manager) typically happen at the beginning of the project, and there wasn't time to cover all the relevant information in the reading / discussion component of the class before the students needed to start playing the DH RPG, given 10-week quarters. The repetition would have the added benefit of giving students exposure to the experience of an ad-hoc project, as a further motivating force for adopting better project management practices the second time around. 
Unfortunately, while two play-throughs of the DH RPG may have been possible with five students and a time limit on turns, the additional players made it impossible. Furthermore, the fact that the turns were open-ended enabled the valuable consultative process around character's experiences. Halfway through the quarter, the characters were only six months into the year of the DH RPG and had not even finished digitizing the materials in their project. As a class, we discussed the pros and cons of "rebooting" the game and being able to make different choices - at the cost of once again only reaching the halfway point. Even though the consequences of less-thanideal early choices were starting to become apparent, the students were committed to seeing the story they had started through to the end.

To mitigate the loss of the game's repetition, there was time at the end of the quarter that was rescheduled for a thorough debrief and discussion, with the expectation that insights about the importance of project management would feature prominently. Instead, the final two class sessions had to be held via Zoom due to the COVID-19 shutdown of Stanford University's campus, with the instructor awkwardly rolling the physical dice in the camera view of her phone, which was logged into Zoom as a separate "DiceCam" participant. None of the numerous online dice roller apps supported the class's particular needs around one differently-coloured die. At that point, both the students and instructor were new to virtual classes. The logistical impediments of Zoom in general, and doing a tabletop RPG via Zoom in particular, further slowed the process of students' turns. The assistant professor character's final D20 roll for tenure happened in the last couple minutes of the final class, leaving little room to process the results, let alone the broader context of the project's trajectory. That same day, Stanford announced that undergraduates should move out and not return after spring break. COVID-19 was admitted into the in-game universe during the final three class sessions by a student vote that was hardly unanimous. But even in a class that focused a great deal of attention on the many factors beyond one's own control in DH projects and the academy writ large, no one anticipated the extent or depth of the disruption from this particularly catastrophic metaphorical dice roll. The loss of time and space to meaningfully debrief at the conclusion of this world we had co-created was devastating. There was no closure for the class as a whole, just an abrupt ending. Individual email exchanges with some of the students afterwards indicated that the whole experience had an impact on them. Some of these reflections evolved into "Academia Is a Dice Roll" in Debates in DH: The Digital Futures of Graduate Study in the Humanities (Backman et al., forthcoming). 


\section{Lessons from the DH RPG}

The DH RPG makes players grapple with the personal and institutional aspects of DH projects: two sets of variables that are often treated as outside the scope of project management, but which can be integral to a project's success or failure. It works best when players have a diverse mix of institutional roles and backgrounds and can consult one another's experiences. Much as in the real world, the range of things that happen in the game is much wider than any taxonomy of scholarly activities. The characters that players create are driven as much by their personalities, life circumstances, and back stories as they are their skill stats. A deadline doesn't guarantee that the character will buckle down and work; they may run off to a cabin in the woods with no internet instead. To avoid foreclosing on any of these possible actions, it is difficult to imagine adapting the DH RPG into a single-player computer version, as dhQuest has done. It is, at its core, intensely interpersonal-much like DH projects themselves.

All of the DH RPG materials are freely available for reuse and adaptation. $\underline{10}$ Its origin in the academia of US-based research institutions is evident and it would require a different set of roles and skills to be successfully translated into other contexts. The game could be incorporated into courses and week-long training events, in order to give students an environment to practice their project management skills-or recognize that they need to develop some. Still, playing the DH RPG requires a significant time commitment, which renders it unfeasible for shorter workshops. While the experiential framing of the game is a major part of its charm and impact, some of the concepts from the game could be incorporated into more traditional project management pedagogical settings, including:

1. The impact of institutional roles and interpersonal relationships on the project's trajectory and decision-making approaches. Ensure that students understand what each of the relevant institutional roles is and means (e.g., assistant vs. an adjunct professor, librarian vs. staff).

2. A frank discussion of participant incentives, how and where they align, and how to navigate situations where they do not. For instance, what does meaningful work look like to a junior faculty member? What are they rewarded for doing, and what do they see as a time suck? How is that different for a librarian?

3. Common loci of ethical dilemmas and strategies for addressing them within the project team. There are many, ranging from labor practices to engagement with the public (particularly minoritized communities) to web scraping public fora. Seek out people with experience navigating some of these situations. 
4. Acknowledging the fact that project participants have complex, challenging lives outside the project, and the impact of those circumstances on the project's timeline and trajectory. Students may not feel comfortable discussing their own personal situation in a classroom context. You can draw upon materials compiled by groups that aim to disrupt academic business-as-usual, such as the Visionary Futures Collective (which brings together humanists across the United States to tackle challenges relating to the present and future of higher education) and DH-WoGeM (Women and Gender Minorities in DH) $\underline{11}, \underline{12}$.

5. The role of the "randomness die" concept, and the importance of developing project management practices that can be nimble and flexible when "things happen." Even without the DH RPG framing, you can give students real scenarios, and discuss how they would respond.

Project management is much more than a set of facts and procedures: it is a practice that must be enacted and is continually shaped and refined by people's experiences with the way that "best practices" can become upended by reality. Empathy and imagination are crucial for successfully navigating those moments where what one "should" do conflicts with what needs to be done in order to do right by the other participants in the project, whether or not you know them by name.

\section{Works Cited}

Backman et al., forthcoming. "Academia is a Dice Roll" in Debates in DH: The Digital Futures of Graduate Study in the Humanities. Eds. Simon Appleford, Gabriel Hankins, and Anouk Lang.

Cowan, T.L., and Jasmine Rault. "Onlining queer acts: Digital research ethics and caring for risky archives." Women \& Performance: A Journal of Feminist Theory. Vol. 28, no. 2, May 2018, pp. 121-142. doi.org/10.1080/0740770X.2018.1473985.

Dombrowski, Quinn. DLCL 205/305: Project Management \& Ethical Collaboration for Humanists. 2019. Standford University, 2020. GitHub, github.com/quinnanya/dlcl205.

Drucker, Johanna, and Geoffrey Rockwell. "Introduction: Reflections on the Ivanhoe game.” TEXT Technology, No. 2, 2003, vii-xviii. doi.org/10.7939/R34M91Q $\underline{30}$.

Fitzpatrick, Kathleen. Generous Thinking: A Radical Appraoch to Saving the University. Johns Hopkins University Press. 2019. 
Handel, Dinah. DLCL class presentation. profiles.stanford.edu/dinah-handel.

Gertz, Genelle. “Genelle Gertz on 'Gaming Paradise Lost'.” DH @W\&L blog.

September 10, 2015. digitalhumanities.wlu.edu/blog/2015/09/10/genelle-gertz-ongaming-paradise-lost.

Kennedy, Kara. “A Long-Belated Welcome: Accepting Digital Humanities Methods into Non-DH Classrooms." DH Quarterly, vol. 11, no 3, October

2017. www.digitalhumanities.org/dhq/vol/11/3/000315/000315.html.

Lord, Gregory, et al. dhQuest. dhquest.dhinitiative.org.

Rehn, Andrea. “Developing a Model: Highlights from DHSI 2015.” Whittier College DigLibArts blog. June 12, 2015. diglibarts. whittier.edu/developing-a-model-highlightsfrom-dhsi-2015/.

Robertson, Tara. “Digitization: just because you can, doesn't mean you should.” Tara Robertson Consulting. March 20, 2016. tararobertson.ca/2016/oob/.

—_ "Update on On Our Backs and Reveal Digital.” Tara Robertson Consulting. August 15, 2016. tararobertson.ca/2016/oob-update/.

[1]

[2] The dhQuest domain name was not renewed in 2018, but as of 2021, a working version of the game was available at http://dhquest.dhinitiative.org $L$, however, the PDFs with instructions for tabletop play are broken links.

[]] https://github.com/gplord/DHQuest

${ }^{[4]}$ It is difficult to find information about dhQuest online. The domain has not been renewed since 2018, and the Wayback Machine captured very little of the previous website, not even the PDFs of the character sheets. Rehn 2015 is one of the few (if brief) accounts of the game, along with a few tweets from Jacob Heil (https://twitter.com/dr heil/status/876819611467100160) and others.

[ㄷ] The zine does not currently have a website, not least because of anti-LGBTQ legislation in Russia, but a number of issues have been posted online by someone in Germany, at https://issuu.com/journal-ostrov. 
[] Depending on the player's choices during character creation, some of those activity points may not be freely allocatable. Having children under the age of 10 comes with a $20 \%$ reduction in available activity points; for children between $10-18$, there is a $10 \%$ reduction.

[7] In D\&D this would be the "dungeon master."

[] An important note about sysadmins: to maintain realism, sysadmins are the only character type able to perform magic in the DH RPG.

[9] All course materials are available as part of the GitHub account https://github.com/dhrpg.

[10] https://visionary-futures-collective.github.io/

[11] http://www.dhwogem.org/

\section{Footnotes}

1. See, for instance, project management as a regular part of DHSI's workshop offerings. $\triangleq$

2. The dhQuest domain name was not renewed in 2018, but as of 2021, a working version of the game was available at http://dhquest.dhinitiative.org $L$, however, the PDFs with instructions for tabletop play are broken links. $\subseteq$

3. https://github.com/gplord/DHQuest $\leftrightharpoons$

4. https://playdhcu5000.github.io/dh-unplugged/ $\triangleq$

5. It is difficult to find information about dhQuest online. The domain has not been renewed since 2018, and the Wayback Machine captured very little of the previous website, not even the PDFs of the character sheets. Rehn 2015 is one of the few (if brief) accounts of the game, along with a few tweets from Jacob Heil (https://twitter.com/dr heil/status/876819611467100160) and others. $\triangleq$

6. The zine does not currently have a website, not least because of anti-LGBTQ legislation in Russia, but a number of issues have been posted online by someone in Germany, at https://issuu.com/journal-ostrov.

7. Depending on the player's choices during character creation, some of those activity points may not be freely allocatable. Having children under the age of 10 
comes with a $20 \%$ reduction in available activity points; for children between $10-18$, there is a $10 \%$ reduction. $\subseteq$

8. In D\&D this would be the "dungeon master."

9. An important note about sysadmins: to maintain realism, sysadmins are the only character type able to perform magic in the DH RPG.

10. All course materials are available as part of the GitHub account https://github.com/dhrpg.

11. https://visionary-futures-collective.github.io/ $\triangleq$

12. http://www.dhwogem.org $L \leftrightharpoons$ 